\title{
Atención Integral de Salud, competencia técnica en personal de la Red Norte y sus factores
}

\author{
Integral Health Care, technical competence in personnel of the
}

North Network and its factors

Atenção Integral de Saúde, competência técnica em pessoal da Rede Norte e seus factores

\section{LUIS ALBERTO CHIHUANTITO ABAL ${ }^{1}$}

\begin{abstract}
RESUMEN
La presente investigación tuvo por objetivo determinar los factores asociados a la competencia técnica del personal de salud en la aplicación de la atención integral de salud en la Red de Servicios de Salud Cusco Norte. Se desarrolló en establecimientos de salud de Cusco, Anta, Calca y Urubamba. La población estuvo conformada por médicos, enfermeras, odontólogos, obstetrices y personal técnico; con un total de 420 personas y una muestra de 120 trabajadores. Se concluye que el nivel de conocimientos acerca de Atención Integral de Salud estaría influido, en el análisis bivariado, por la profesión u ocupación, condición laboral y capacitación; y al análisis multivariado por la condición laboral, tiempo de servicio y capacitación; así mismo que las prácticas de Atención Integral de Salud estaría influida, en el análisis bivariado, por la edad, sexo, condición laboral y capacitación; y al análisis multivariado por el sexo, condición laboral y capacitación.
\end{abstract}

Palabras clave: atención integral; salud; nivel de conocimientos; servicio de salud.

\begin{abstract}
The objective of this research was to determine the factors associated with the technical competence of health personnel in the application of integrated health care in the North Cusco Health Services Network. It was developed in health centers in Cusco, Anta, Calca and Urubamba. The population was made up of doctors, nurses, dentists, midwives and technical staff with a total of 420 people and a sample of 120 workers. It is concluded that the level of knowledge about Integral Health Care would be influenced by the bivariate analysis by profession or occupation, working condition and training; and the multivariate analysis by working condition, time of service and training. Likewise, Integral Health Care practices would be influenced in the bivariate analysis by age, sex, labor condition and training; and in the multivariate analysis by sex, labor condition and training.
\end{abstract}

Keywords: integral attention; health; level of knowledge; health service.

1. UNIVERSIDAD ANDINA DEL CUSCO, Perú | Ichihuantito@uandina.edu.pe | https://orcid.org/0000-0002-3340-981X 


\section{RESUMO}

O objectivo desta investigação era determinar os factores associados à competência técnica do pessoal de saúde na aplicação de cuidados de saúde integrados na Rede de Serviços de Saúde do Norte de Cusco. Foi realizado em instalações sanitárias em Cusco, Anta, Calca e Urubamba. A população era composta por médicos, enfermeiros, dentistas, parteiras e pessoal técnico com um total de 420 pessoas e uma amostra de 120 trabalhadores. Conclui-se que o nível de conhecimento sobre cuidados de saúde abrangentes seria influenciado pela análise bivariada por profissão ou ocupação, condição de trabalho e formação; e a análise multivariada por condição de trabalho, tempo de serviço, e formação. Do mesmo modo, as práticas de Cuidados de Saúde Integrados seriam influenciadas na análise bivariada por idade, sexo, estatuto profissional e formação; e na análise multivariada por sexo, estatuto profissional e formação.

Palavras-chave: atenção Integral; saúde; nível de conhecimentos; serviço de saúde.

\section{INTRODUCCIÓN}

La Atención Integral de Salud es la provisión continua y con calidad de una atención orientada hacia la promoción, prevención, recuperación y rehabilitación en salud para las personas, en el contexto de su familia y comunidad. (Ministerio de Salud, 2016).

Según González et al. (2019) la provisión de servicios de salud y la atención integral al individuo, la familia y la comunidad exigen de cambios acordes con las estrategias sanitarias, en los que se comprendan los objetivos del sector en su relación con la política social, educacional y científico-tecnológica del país, tríada programática encaminada a crear las bases para un proyecto nacional más justo y equitativo con la vista puesta en el criterio del desarrollo de la sociedad, visualizando el cuidado a las personas sanas o enfermas como un eje estratégico para el desarrollo de la nación.

El Modelo de Atención es una construcción social que sustenta una respuesta individual o colectiva a una o más necesidades de salud (práctica social en salud).

En tal sentido, un modelo de atención de salud describe el tipo de respuesta de salud que la sociedad desea y decide sostener y recibir. Es definida como el marco conceptual de referencia que define el conjunto de políticas, sistemas, procesos e instrumentos que, operando coherentemente, garantizan la atención a la persona, la familia y la comunidad, para satisfacer sus necesidades de salud (necesidades reales que son percibidas o no por la población).

No hay modelo malo, cada modelo responde a una determinada realidad y en una determinada época.

En la actualidad, luego de más de dos décadas del modelo previo y a pesar de los logros que pudieran haberse dado se han evidenciado señales de agotamiento que indican la necesidad de un cambio (Ministerio de Salud, 2001). 
Este Modelo busca mejorar las capacidades de los profesionales de la salud mejorando su disposición y aptitudes en el trabajo.

Una herramienta prioritaria durante la ejecución de este modelo, es incentivar acciones entre varios sectores para actuar directamente sobre los determinantes sociales de la Salud que recaen sobre la persona, la familia y la comunidad. Además, es importante el control y liderazgo adecuado de las autoridades sanitarias para mejorar la oferta de salud, las acciones entre sectores e involucrar a la comunidad en esto (Ministerio de Salud, 2011). Según reporte de la Dirección Regional de Salud Cusco (2019), dicha atención está a cargo de personal de salud competente a partir de un enfoque biopsicosocial.

Sin embargo, en la Red Cusco Norte aún existe personal de salud que desconoce la propuesta y persiste con actitudes y prácticas desfavorables en su aplicación. Quizá la insuficiente capacitación recibida en este aspecto, sumado a la condición laboral, tiempo de servicios

y otros factores contribuyan a la persistencia del problema. Por tanto, se hace necesaria la identificación de los determinantes que repercuten en la aplicación de dicha política de salud, la misma que permitirá plantear una serie de estrategias que coadyuven al acceso de una atención de calidad.

El presente estudio tuvo por objetivo determinar los factores que influyen en la competencia técnica del personal de salud en la aplicación de la Atención Integral de Salud en la Red de Servicios de Salud Cusco Norte.

\section{METODOLOGÍA}

La población de la cual se obtuvo la muestra, estuvo conformada por 480 trabajadores entre médicos, enfermeras, odontólogos, obstetrices y personal técnico de las provincias de Cusco, Anta, Calca y Urubamba pertenecientes a la Red de Servicios de Salud Cusco Norte. El Muestreo fue no probabilístico intencionado, mediante el cual, a partir del área de estudio; se identificaron establecimientos de salud que cumplieron con la característica de ser Centros de Salud, por contar éstos con equipo de salud completo, obteniéndose una muestra final de 120 unidades muestrales.

Los instrumentos para la recolección de datos fueron; una encuesta estructurada y una guía de observación, la encuesta identificó características generales y midió el nivel de conocimientos sobre la Atención Integral de Salud mediante diez preguntas con alternativas múltiples, en las dimensiones de conceptos generales, componentes, principios, etc. ; para su calificación se utilizó la escala vigesimal, cuya expresión final fue : Deficiente (De 0 a 10 puntos), Regular (De 11 a 13 puntos), Bueno (De 14 a 16 puntos), Muy Bueno (De 17 a 18 puntos) y Sobresaliente (De 19 a 20 puntos). La guía de observación permitió calificar 
las prácticas desarrolladas durante las consultas, de acuerdo al Manual de implementación del Modelo de Atención Integral de Salud en las dimensiones de aplicación de instrumentos, calificación de riesgos, sistema de referencia y contrareferencia, entornos saludables y uso de estrategias; la guía estuvo conformada por 30 ítems, cuyo valor asignado a cada uno de ellos fue de 0 a 30 puntos, lo que finalmente permitió categorizar a las prácticas como: Adecuada (De 21 a 30 puntos), Parcialmente adecuada (De 11 a 20 puntos) e Inadecuada (De 0 a 10 puntos).

Los instrumentos fueron mejorados a partir de los resultados de una Prueba piloto dirigida al $10 \%$ de la muestra, en la Red de Servicios de Salud Cusco Sur, por contar con una población con similares características a las de la muestra. La aplicación de los instrumentos se realizó previa autorización de la Dirección de la Red Cusco Norte.

Para el procesamiento de la información se utilizó el programa SPSS, al cual se vaciaron los datos. En cuanto al análisis estadístico se utilizó el Chi cuadrado, rho de Spearman y Regresión logística; pruebas estadísticas que permitieron relacionar las Variables Independientes con la Dependiente.

\section{RESULTADOS Y DISCUSIÓN}

\section{Características generales}

La Tabla $\mathrm{N}^{\circ} 1$, muestra las características del personal participante, donde se observa que más de la tercera parte tenía entre 30 y 39 años; seguida de 20 a 29 y 40 a 49 años con la misma frecuencia (27,5\%), podemos referir que el 94,17\% tenía entre 20 a 49 años de edad. Asimismo, se observa cierto predominio del sexo femenino, la mayor frecuencia (cuarta parte) eran médicos, seguido de enfermeros y obstetrices en la misma frecuencia. Este resultado coincide con lo publicado en la Revista Ciencia y Enfermería de Brasil por Harrison et al. (2005), donde se encontró que la fuerza de trabajo de salud es eminentemente femenina y de edad inferior a 40 años.

Se encontró poca disponibilidad del personal de salud a ser evaluado, por el temor de que la intervención tenga carácter punitivo. Esta limitación se pudo superar mediante la explicación previa sobre el propósito del estudio.

La edad media fue de 30,05 años, con una moda de 31 y una edad mínima de 20 y máxima de 61. En esta tabla también tenemos que el tiempo medio de servicio fue de 8 años con un mínimo de 1 y máximo de 24 . Este resultado no coincide con la investigación de Juárez-Adauta (2012) quienes dan a conocer que la edad de la población de trabajadores de salud tiene una media de 35 años. 
Tabla $\mathrm{N}^{\circ} 1$.

Características del personal participante

\begin{tabular}{|c|c|c|}
\hline CARACTERÍSTICAS & $N^{\circ}=120$ & $\%=100.00$ \\
\hline \multicolumn{3}{|l|}{ a) Edad (años) } \\
\hline $20-29$ & 33 & 27,50 \\
\hline $30-39$ & 47 & 39,17 \\
\hline $40-49$ & 33 & 27,50 \\
\hline $50-59$ & 6 & 5,00 \\
\hline $60 \mathrm{a}+$ & 1 & 0,83 \\
\hline \multicolumn{3}{|l|}{ b) Sexo } \\
\hline Masculino & 52 & 43,33 \\
\hline Femenino & 68 & 56,67 \\
\hline \multicolumn{3}{|l|}{ c) Profesión u Ocupación } \\
\hline Médico & 30 & 25,00 \\
\hline Enfermero(a) & 24 & 20,00 \\
\hline Obstetriz & 24 & 20,00 \\
\hline Odontólogo & 21 & 17,50 \\
\hline Técnico & 21 & 17,50 \\
\hline \multicolumn{3}{|l|}{ d) Condición Laboral } \\
\hline Nombrado & 35 & 29,17 \\
\hline Contratado & 67 & 55,83 \\
\hline Serum & 18 & 15,00 \\
\hline \multicolumn{3}{|c|}{ e) Tiempo de Servicios (años) } \\
\hline$<5$ & 58 & 48,33 \\
\hline $5-10$ & 17 & 14,17 \\
\hline $11-15$ & 29 & 24,17 \\
\hline $16-20$ & 10 & 8,33 \\
\hline$>20$ & 6 & 5,00 \\
\hline \multicolumn{3}{|l|}{ f) Capacitación en AIS } \\
\hline Sí & 77 & 64,17 \\
\hline $\mathrm{NO}$ & 43 & 35,83 \\
\hline
\end{tabular}

\section{Factores que influyen en el nivel de conocimiento}

La Tabla $N^{\circ}$ 2, muestra la influencia de la Edad, Sexo, Profesión/Ocupación, Condición laboral, Tiempo de Servicios y Capacitación en el Nivel de Conocimientos sobre la Atención Integral en Personal de Salud de la Red Cusco Norte, donde se observa que cerca de las tres cuartas partes $(73,33 \%)$ de los encuestados cuentan con un Nivel de Conocimientos Deficiente sobre Atención Integral de Salud, seguido del 15\% con un nivel de conocimientos bueno y un $11,67 \%$ con un nivel de conocimientos regular. No se encontraron casos de nivel "Muy Bueno" ni "Sobresaliente". Este resultado es similar a lo encontrado por Rodríguez et al (2001) en su estudio sobre Educación basada por competencias en personal de salud, 
donde el nivel de conocimiento del personal de salud como médicos y enfermeras, en términos generales es similar en los dos grupos, una fracción mínima de médicos y enfermeras tiene información o conocimiento sobre la totalidad de los conceptos. Solo $44 \%$ conocen parcialmente y más de la mitad de la población estudiada tiene mínimo conocimiento sobre estos conceptos. Por otro lado, los resultados de la presente investigación, se contraponen al estudio desarrollado por Ochoa-Alencastre et al. (2009) quienes a la evaluación del personal de salud del primer nivel de atención en el 2005, encuentran que una proporción cercana a la mitad de los encuestados muestra un nivel deficiente de conocimientos acerca de Atención Integral de Salud, la quinta parte de estos presenta un nivel regular, casi uno de cada cinco profesionales de la salud tienen buen nivel y el $15 \%$ tienen un desempeño muy bueno.

En cuanto a la influencia de ciertas características del personal sobre su nivel de conocimientos, vemos que mientras la edad se incrementa, la frecuencia del personal con un nivel "Deficiente" también se incrementa, llegando al 100\% de los que tenían 50 a más años de edad. El nivel "Regular" fue más frecuente a menor edad (20 -29 años) y el nivel "Bueno" fue más frecuente, más de un tercio, de los 40 a 49 años de edad, seguido de 20 a 29 años, a la prueba estadística bivariada encontramos que la edad no sería un factor que influencia en el nivel de conocimientos acerca de Atención Integral de Salud.

Los trabajadores de sexo masculino son los que en más de las dos terceras partes tuvo un nivel "Deficiente" en comparación con el femenino donde este nivel alcanzó el 70,59\%; en el nivel "Regular", en cerca de 3 veces más frecuente fue el sexo femenino; y el nivel "Bueno" fue 4,07\% más frecuente entre los varones; sin embargo, al análisis bivariado, no se encontró que el sexo tuviera influencia.

Finalmente, la Tabla $\mathrm{N}^{\circ} 2$ muestra que en la totalidad de Odontólogos(as) y Técnicos(as) que tuvieron un nivel "Deficiente" y los Enfermeros(as) y Médicos(as); los que tendrían mejor nivel alcanzado al calificativo de "Bueno" sobre todo en el primer grupo profesional referido; al análisis bivariado se encontró que la profesión u ocupación si influencia en el nivel de conocimientos acerca de Atención Integral de Salud.

En las tres condiciones laborales, en más de las dos terceras partes del personal estudiado tuvo un nivel de conocimientos "Deficiente" sobre todo el personal SERUM; siendo los nombrados los que en mayor frecuencia (33,33\%) alcanzaron un nivel de "Bueno"; al análisis bivariado se encontró que la condición laboral si sería un factor que influenciaría en el nivel de conocimientos.

En cuanto al tiempo de servicios, se encontró que, en más de la mitad de todas las categorías, incluso en algunos casos hasta el 100\% tuvieron un nivel "Deficiente"; y los que tenían 11 a 15 años de servicios son los que en mayor frecuencia $(41,38 \%)$ alcanzaron un nivel "Bueno"; sin embargo, no se encontró que esta condición (tiempo de servicio) influyera en el nivel de conocimientos.

Del personal que no recibió capacitación, la gran mayoría (93,02\%) tuvo un nivel "Deficiente" en comparación con los que sí la recibieron; donde el 62,34\% alcanzó este nivel y el 19,48\% de "Bueno". Siendo al análisis bivariado, la capacitación un factor que influiría. 
Al análisis multivariado se encontró que la condición laboral, el tiempo de servicio y la capacitación son factores que influyen en el nivel de conocimientos sobre Atención Integral de Salud.

\section{Tabla $\mathrm{N}^{\circ} 2$.}

Influencia de la edad, sexo y profesion/ocupacion, condición laboral, tiempo de servicio y capacitación en el nivel de conocimientos sobre atención integral del personal de la red de Servicios de Salud Cusco Norte

\begin{tabular}{|c|c|c|c|c|c|}
\hline \multirow{2}{*}{\multicolumn{2}{|c|}{ CARACTERÍSTICAS }} & \multicolumn{4}{|c|}{ NIVEL DE CONOCIMIENTOS } \\
\hline & & \multirow{2}{*}{$\begin{array}{l}\text { Total }=120 \\
N^{\circ}\end{array}$} & \multirow{2}{*}{$\begin{array}{l}\text { Deficiente }=88 \\
N^{0}(\%)\end{array}$} & \multirow{2}{*}{$\begin{array}{l}\text { Regular }=14 \\
N^{0}(\%)\end{array}$} & \multirow{2}{*}{$\begin{array}{l}\text { Bueno = } 18 \\
N^{0}(\%)\end{array}$} \\
\hline & & & & & \\
\hline \multirow{5}{*}{ a) Edad (años) } & $20-29$ & 33 & $23(69,70)$ & $7(21,21)$ & $3(9,09)$ \\
\hline & $30-39$ & 47 & $37(78,72)$ & $7(14,89)$ & $3(6,38)$ \\
\hline & $40-49$ & 33 & $21(63,64)$ & - & $12(36,36)$ \\
\hline & $50-59$ & 6 & $6(100,00)$ & - & - \\
\hline & $\geq 60$ & 1 & $1(100,00)$ & - & - \\
\hline \multicolumn{3}{|c|}{ rho de Spearman $=0,030$} & \multicolumn{2}{|c|}{$\mathrm{p}=0,744$} & \\
\hline \multirow{2}{*}{ b) Sexo } & Masculino & 52 & $40(76,92)$ & $3(5,77)$ & $9(17,31)$ \\
\hline & Femenino & 68 & $48(70,59)$ & $11(16,18)$ & $9(13,24)$ \\
\hline \multicolumn{2}{|c|}{$X^{2}=1,944$} & \multicolumn{2}{|l|}{$\mathrm{p}=0,378$} & & \\
\hline \multirow{5}{*}{$\begin{array}{l}\text { c) Profesión/ } \\
\text { Ocupación }\end{array}$} & Médico & 30 & $18(60,00)$ & $3(10,00)$ & $9(30,00)$ \\
\hline & Enfermero(a) & 24 & $15(62,50)$ & - & $9(37,50)$ \\
\hline & Obstetriz & 24 & $13(54,17)$ & $11(45,83)$ & - \\
\hline & Odontólogo & 21 & $21(100,00)$ & - & - \\
\hline & Técnico & 21 & $21(100,00)$ & - & - \\
\hline \multicolumn{2}{|c|}{$X^{2}=52,650$} & \multicolumn{3}{|l|}{$\mathrm{p}=0,000$} & \\
\hline \multirow{3}{*}{$\begin{array}{l}\text { d) Condición La- } \\
\text { boral }\end{array}$} & Nombrado & 36 & $24(66,67)$ & - & $12(33,33)$ \\
\hline & Contratado & 66 & $49(74,24)$ & $14(21,21)$ & $3(4,55)$ \\
\hline & Serum & 18 & $15(83,33)$ & - & $3(16,67)$ \\
\hline \multicolumn{2}{|c|}{$X^{2}=23,273$} & \multicolumn{2}{|l|}{$\mathrm{p}=0,000$} & & \\
\hline \multirow{5}{*}{$\begin{array}{l}\text { e) Tiempo de } \\
\text { servicio } \\
\text { (años) }\end{array}$} & $<5$ & 58 & $41(70,69)$ & $14(24,14)$ & $3(5,17)$ \\
\hline & $5-10$ & 17 & $17(100,00)$ & - & - \\
\hline & $11-15$ & 29 & $17(58,62)$ & - & $12(41,38)$ \\
\hline & $16-20$ & 10 & $7(70,00)$ & - & $3(30,00)$ \\
\hline & $>20$ & 6 & $6(100,00)$ & - & - \\
\hline \multicolumn{2}{|c|}{ Rho de Spearman $=0,85$} & $\mathrm{p}=0$ & & & \\
\hline \multirow{2}{*}{ f)Capacitación } & $\mathrm{Si}$ & 77 & $48(62,34)$ & $14(18,18)$ & $15(19,48)$ \\
\hline & No & 43 & $40(93,02)$ & - & $3(6,98)$ \\
\hline \multicolumn{2}{|c|}{$X^{2}=11,868$} & $\mathrm{p}=0,003$ & & & \\
\hline
\end{tabular}




\section{Factores que influyen en la práctica}

La Tabla N 3, muestra la influencia del Grupo etario, Sexo, Profesión/Ocupación, Condición laboral, Tiempo de Servicio y Capacitación en las prácticas de Atención Integral de Salud en personal de la Red de Servicios de Salud Cusco Norte, donde se observa que, de cada 10 trabajadores de salud, aproximadamente uno tendría una práctica Adecuada, cuatro Parcialmente Adecuada y cinco Inadecuada. En cuanto a la edad, podemos referir que entre los que tuvieron una práctica calificada como Inadecuada, en mayor frecuencia estaban los que tenían entre 30 a 39 años, seguida de 20 a 29 años; y en edades mayores (a partir de los 50 años) no se encontró casos donde la práctica fuera Inadecuada; lo que sí se observó en estos grupos de edad, la frecuencia más alta de prácticas calificadas como "Adecuadas". Al análisis bivariado encontramos que la edad si influye en las prácticas.

Las prácticas adecuadas en mayor frecuencia estuvieron entre los de sexo masculino en casi cinco veces más que en los de sexo femenino; y las prácticas inadecuadas fueron discretamente mayor en frecuencia entre los de sexo masculino. Al análisis bivariado, el sexo si influye.

En las prácticas adecuadas, en mayor porcentaje eran enfermeros(as) seguido de médicos; y en las prácticas inadecuadas en mayor frecuencia estaban las Obstetrices seguida de odontólogos; sin embargo, no se encontró influencia de la profesión u ocupación en las prácticas de Atención Integral de Salud.

El nivel de prácticas "Inadecuadas" en mayor frecuencia se da entre el personal SERUM seguido del Contratado; en el nivel "Parcialmente Adecuado", los nombrados están con más de la mitad seguida del personal contratado; y en el nivel "Adecuado", la mayor frecuencia correspondió al personal SERUM, seguido del contratado; siendo que al análisis bivariado habría influencia de la condición laboral en las prácticas acerca de Atención Integral.

Entre los que alcanzaron un calificativo de prácticas "Inadecuadas", el mayor porcentaje fue entre 5 a 10 años, seguido con la misma frecuencia en menos de 5 años y de 11 a 15 años. En los que tendrían prácticas "Parcialmente Adecuadas" estuvo el 100\% de los que tenían más de 20 años de servicio; seguido de 16 a 20 años; y con prácticas "Adecuadas" en mayor frecuencia los que tenían entre 11 a 15 años de servicio, seguido de los de menor de 5 años, no encontrándose influencia. En lo que respecta a capacitación entre los que tenían prácticas "Inadecuadas" predominaron los que no recibieron capacitación; entre los que tenían "Parcialmente Adecuada" la frecuencia fue muy semejante en ambos grupos y entre los que tenían prácticas "Adecuadas" predominaron los que recibieron capacitación; encontrándose que el recibir capacitación o no influiría en el nivel de prácticas acerca de Atención Integral de Salud.

Al hacer el análisis multivariado encontramos que los factores que influirían en las prácticas acerca de Atención Integral de Salud serían la edad, el sexo, la condición laboral, el tiempo de servicio y capacitación. 
Tabla $\mathrm{N}^{\circ} 3$.

Influencia de la edad, sexo y profesion/ocupacion, condición laboral, tiempo de servicio y capacitación en las practicas del personal participante de la Red de Servicios de Salud Cusco Norte

\begin{tabular}{|c|c|c|c|c|c|c|}
\hline & & & \multicolumn{4}{|c|}{ PRÁCTICAS } \\
\hline & & & Total $=120$ & Adecuada $=15$ & $\begin{array}{l}\text { Parcialmente Ade- } \\
\text { cuada }=47\end{array}$ & $\begin{array}{l}\text { Inadecua- } \\
\mathrm{da}=\mathbf{5 8}\end{array}$ \\
\hline \multicolumn{3}{|c|}{ CARACTERÍSTICAS } & $\mathbf{N}^{\mathbf{0}}$ & $N^{0}(\%)$ & $\mathbf{N}^{0}(\%)$ & $\mathbf{N}^{0}(\%)$ \\
\hline \multirow{5}{*}{$\begin{array}{l}\text { a) Grupo etá- } \\
\text { reo }\end{array}$} & 20 & 29 & 33 & $6(18,18)$ & $9(27,27)$ & $18(54,55)$ \\
\hline & 30 & 39 & 47 & $2(4,26)$ & $14(29,79)$ & $31(65,96)$ \\
\hline & 40 & 49 & 33 & $3(9,09)$ & $21(63,64)$ & $9(27,27)$ \\
\hline & 50 & 59 & 6 & $3(50,00)$ & $3(50,00)$ & - \\
\hline & $\geq 6$ & & 1 & $1(100,00)$ & - & - \\
\hline \multicolumn{3}{|c|}{ Rho de Spearman $=-0,253$} & \multicolumn{2}{|c|}{$\mathrm{p}=0,005$} & & \\
\hline \multirow{2}{*}{ b) Sexo } & Ma & sculino & 52 & $12(23,08)$ & $14(26,92)$ & $26(50,00)$ \\
\hline & Fen & nenino & 68 & $3(4,41)$ & $33(48,53)$ & $32(47,06)$ \\
\hline \multicolumn{3}{|c|}{$\mathrm{X}^{2}=11,121$} & \multicolumn{2}{|l|}{$\mathrm{p}=0,004$} & & \\
\hline \multirow{5}{*}{$\begin{array}{l}\text { c) Profesión / } \\
\text { Ocupación }\end{array}$} & Mé & dico & 30 & $5(16,67)$ & $11(36,67)$ & $14(46,67)$ \\
\hline & Enf & ermero(a) & 24 & $5(20,83)$ & $8(33,33)$ & $11(45,83)$ \\
\hline & Ob & tetriz & 24 & $2(8,33)$ & $9(37,50)$ & $13(54,17)$ \\
\hline & Od & pntólogo & 21 & $3(14,29)$ & $7(33,33)$ & $11(52,38)$ \\
\hline & Téc & nico & 21 & - & $12(57,14)$ & $9(42,86)$ \\
\hline \multicolumn{3}{|c|}{$X^{2}=12,445$} & \multicolumn{2}{|l|}{$\mathrm{p}=0,132$} & & \\
\hline \multirow{3}{*}{\multicolumn{2}{|c|}{$\begin{array}{l}\text { d) Condición La- } \\
\text { boral }\end{array}$}} & Nombrado & 35 & $3(8,57)$ & $20(57,14)$ & $12(34,29)$ \\
\hline & & Contratado & 67 & $9(13,43)$ & $24(35,82)$ & $34(50,75)$ \\
\hline & & Serum & 18 & $3(16,67)$ & $3(16,67)$ & $12(66,67)$ \\
\hline \multicolumn{3}{|c|}{$X^{2}=9,511$} & \multicolumn{2}{|l|}{$\mathrm{p}=0,050$} & & \\
\hline \multirow{5}{*}{\multicolumn{2}{|c|}{$\begin{array}{l}\text { e) Tiempo de } \\
\text { servicio (años) }\end{array}$}} & $<5$ & 58 & $6(10,34)$ & $21(36,21)$ & $31(53,45)$ \\
\hline & & $5-10$ & 17 & $6(35,29)$ & $3(17,65)$ & $8(47,06)$ \\
\hline & & $11-15$ & 29 & $3(10,34)$ & $10(34,48)$ & $16(55,17)$ \\
\hline & & $16-20$ & 10 & - & $7(70,00)$ & $3(30,00)$ \\
\hline & & $>20$ & 6 & - & $6(100,00)$ & - \\
\hline \multicolumn{3}{|c|}{ Rho de Spearman $=-0,060$} & \multicolumn{2}{|c|}{$\mathrm{p}=0,517$} & & \\
\hline \multirow{3}{*}{\multicolumn{2}{|c|}{ f) Capacitación }} & $\mathbf{S i}$ & 77 & $3(3,90)$ & $30(38,96)$ & $45(58,44)$ \\
\hline & & No & 43 & $12(27,91)$ & $17(39,53)$ & $13(30,23)$ \\
\hline & & $X^{2}=1$ & 357 & $\mathrm{p}=0,000$ & & \\
\hline
\end{tabular}




\section{CONCLUSIONES}

El nivel de conocimiento acerca de Atención Integral de Salud está influenciado, en el análisis bivariado, por la profesión u ocupación, condición laboral y capacitación; y al análisis multivariado por la condición laboral, tiempo de servicio y capacitación. Los resultados muestran la existencia de grupos de profesionales que no están comprometidos con la estrategia, a ello se puede agregar que existen solo algunos grupos profesionales que cuentan con la competencia técnica para poder ofrecerla. Las condiciones laborales en el sector salud son precarias, caracterizadas por bajas remuneraciones y pocos estímulos frente a la recargada labor que no permite el desarrollo profesional ni la auto instrucción. El desconocimiento de la esencia de la Atención Integral repercute en el compromiso que tienen cada uno de los profesionales y por consiguiente en su ejecución. El rol que desempeña cada uno de los integrantes del sistema, es muy importante para poder ejecutar las acciones encaminadas al cuidado de la salud, su desconocimiento constituye un riesgo.

Las prácticas de Atención Integral de Salud están influenciadas, en el análisis bivariado, por la edad, sexo, condición laboral y capacitación; y al análisis multivariado por el sexo, condición laboral y capacitación. Los resultados muestran una realidad que se encuentra presente en los establecimientos de salud, caracterizada por personal de salud donde solo 1 de 10 profesionales practica adecuadamente los principios de la atención integral afectando la provisión continua y con calidad de la atención de salud principalmente los cuidados esenciales orientados hacia la promoción, prevención, recuperación y rehabilitación en salud para las personas, en el contexto de su familia y comunidad.

\section{REFERENCIAS}

Dirección Regional de Salud - Cusco. (2019). Avances del Modelo de Atención Integral de Salud. Dirección Regional de Salud Cusco.

González, C. G., Herrera, Y. R., \& Pulgar, G. E. B. (2019). Modelo de atención integral de salud vs. calidad asistencial en el primer nivel, Riobamba 2014-2017. Educación Médica, 20, 136-142. https://doi.org/10.1016/j.edumed.2017.10.023

Harrison, L., Ray Hernandez, A., Cianelli, R., Rivera, M. S., \& Urrutia, M. (2005). Competencias en investigación para diferentes niveles de formación de enfermeras: una perspectiva latinoamericana. Ciencia y Enfermería, 11(1), 59-71. https://doi. org/10.4067/S0717-95532005000100007

Juárez-Adauta, S. (2012). Clima organizacional y satisfacción laboral. Revista médica del instituto Mexicano del Seguro Social, 50(3), 307-314. https://www.medigraphic. $\mathrm{com} / \mathrm{cgi}$-bin/new/resumen.cgi?IDARTICULO=38964

Ministerio de Salud (2016). Implementación del nuevo Modelo de Atención Integral de Salud (MAIS). MINSA 2016.

Ministerio de Salud. (2001). Lineamientos de Política Sectorial para el Periodo 2002 - 2012. MINSA 2001. https://cdn.www.gob.pe/uploads/document/file/417352/344744252836504234520191106-32001-1nhnkg.pdf 
Ministerio de Salud. (2011). Modelo de Atención Integral de Salud Basado en Familia y Comunidad. MINSA 2011. https://determinantes.dge.gob.pe/archivos/1880.pdf

Ochoa-Alencastre, Mercedes, Arnao-Farfán, Cinthia, \& Sanabria-Rojas, Hernán. (2009). Conocimiento sobre metodología educativa para la capacitación de adultos del personal de salud del primer nivel de atención, Perú 2005. Revista Peruana de Medicina Experimental y Salud Publica, 26(1), 27-34. http://www.scielo.org.pe/scielo.php?script=sci_arttext\&pid=S1726-46342009000100006\&lng=es\&tlng=es

Rodríguez, M. C., Ortuño, A. A., Hernández, M. A., \& Moreno, S. P. (2001). Educación Basada en Competencias Educación Basada en Competencias ¿Necesario el conocimiento en el personal de ¿Necesario el conocimiento en el personal de salud? Rev Enferm IMSS, 9(3), 133-136. http://revistaenfermeria.imss.gob.mx/editorial/index. php/revista_enfermeria/article/download/787/746 